Results 65 cases of abdominal TB were identified. Average age was 42 years (range 18-97). 49.2\% females and 50.8\% males. Ethnicity and mean ages are outlined in Table 1.

The mean age of European Caucasians was significantly older than from combined black and minority ethnic (BME) groups (72.4 v 39.5, $\mathrm{p}=0.016)$. The number of cases over the 10 year period has remained stable, with an average of 6.5 per year (range 4-10). 13.85\% were HIV positive. all in BME patients (1 South Asian, 8 Africans).

Conclusion Abdominal TB remains an active disease in London, affecting a wide range of ages and ethnicities, with the majority of patients South Asian and African, in accordance with national data. It should be considered as a differential in all patients, but particularly those who are at high risk. It presents as a disease of elderly Europeans and young BME groups. A significant number of BME group patients have HIV infection.

Disclosure of Interest None Declared.

\section{PTU-091 ANTI-TNF THERAPY REDUCES IONISING RADIATION EXPOSURE IN PATIENTS WITH CROHN'S DISEASE}

D Aggarwal, JK Limdi*. Gastroenterology, Pennine Acute Hospitals NHS Trust, Manchester, UK

\subsection{6/gutjnl-2014-307263.165}

Introduction Patients with Crohn's Disease [CD] are often exposed to ionising radiation for the diagnosis and evaluation of disease with inherent risks from protracted exposure. Meanwhile, bolder definitions of disease control have changed treatment paradigms with earlier introduction of biological therapy in many. Our aim was to compare the effective radiation dose a year prior and 1 and 3 years after initiating anti-TNF therapy or corticosteroid.

Methods We performed a retrospective review of CD patients treated with anti-TNF (infliximab or adalimumab) or corticosteroids at our institution from 2005 to 2013. Clinical data (demographics, disease characteristics, treatment) were obtained from patient records. All instances of imaging in the previous year and 1 and 3 years after initiation of therapy were recorded. Effective and cumulative radiation doses were calculated from published tables [Royal College of Radiologists, UK].

Results We analysed 170 patients with CD (114 anti-TNF, 56 corticosteroid). In the anti-TNF group, $55 \%$ were female (median age 35 yrs;mean disease duration 8.2yrs). Disease location was ileal (46\%), colonic (21\%), ileocolonic (31\%) and perianal $(22 \%)$ with inflammatory, stricturing and penetrating disease in $63 \%, 14 \%$ and $23 \%$ respectively. In the corticosteroid group, $53 \%$ were females (median age 48 ; mean disease duration 13.2yrs). Disease location was ileal (44\%), colonic (27\%), ileocolonic $(29 \%)$ and perianal $(14 \%)$ with inflammatory, stricturing and penetrating disease in $75 \%, 20 \%$ and $5 \%$ respectively.

The anti-TNF cohort had a significant reduction in the cumulative radiation dose $(4.2$ vs. $1.8 \mathrm{mSv}, \mathrm{p}<0.0001)$ compared to the previous year. There was no change in the cumulative radiation dose in the corticosteroid group (7.5 vs.7.3 $\mathrm{mSv}, \mathrm{p}=0.8$ ). The number of CT scans reduced from 3.3 to $1.2(\mathrm{p}<0.0001)$ in the anti-TNF cohort. There was no reduction the number of CT scans in the corticosteroid group after one year $(2.7$ vs. 2.5, $\mathrm{p}=0.006$ ).

In 31 patients with 3-year exposure to corticosteroids, there was a significant increase in the cumulative radiation dose $(7.0$ vs. $13.8 \mathrm{mSv}, \mathrm{p}<0.0003)$ compared to the anti-TNF group $(3.5$ vs. $4.8 \mathrm{mSv}, \mathrm{p}=0.7)$. There was a significant increase in the number of CT scans in the corticosteroid group (2.6 vs. $4.9, \mathrm{p}<$ $0.001)$ compared to the anti-TNF group (2.5 vs. $2.8, \mathrm{p}=0.9)$.

Linear regression analysis showed a decrease in cumulative radiation dose by $2.7 \mathrm{mSv}(\mathrm{p}=0.07)$ and number of CT scans by $2(p<0.001)$ in the anti-TNF group compared to the corticosteroid group within a year of therapy after adjusting for age, gender, disease duration, disease location and disease behaviour.

Conclusion Anti-TNF but not corticosteroid therapy is associated with a significant reduction in diagnostic radiation exposure a year after treatment and persisted after 3 years although not statistically significant

Disclosure of Interest None Declared.

\section{PTU-092 PATIENT AND PROFESSIONALS PERCEPTIONS OF TRAVEL BEHAVIOUR IN INFLAMMATORY BOWEL DISEASE}

${ }^{1} \mathrm{~J}$-P Mulligan*, ${ }^{2} \mathrm{~K}$ Greveson, ${ }^{2} \mathrm{~T}$ Shepherd, ${ }^{2} \mathrm{M}$ Hamilton, ${ }^{2} \mathrm{CD}$ Murray. ${ }^{1}$ University College London Medical School, University College London, UK; ${ }^{2}$ Centre for Gastroenterology, Royal Free Hospital London NHS Foundation Trust, London, UK

\subsection{6/gutjpl-2014-307263.166}

Introduction Travellers with inflammatory bowel disease (IBD) are at greater risk of travel-related morbidity. ${ }^{1}$ ECCO recommend patients seek expert advice prior to travel, including information on vaccination and obtaining antibiotics for selftreatment of travellers diarrhoea. ${ }^{2}$ Wasan et al. report only $3.5 \%$ of patients on immunosuppression therapy were counselled on avoiding particular live vaccines ${ }^{3}$ and $30 \%$ of gastroenterologists would erroneously recommend live vaccines. ${ }^{4}$

Methods We explored both patient and gastroenterology health care professionals (HCP) perceptions of IBD and travel: whether disease affected travel, interventions people took to travel, and whether ECCO guidelines were being followed. IBD patients attending our IBD clinic during November 2013 were asked to complete a questionnaire collecting demographic, disease specific and travel related information. Using N-ECCO and RCN IBD nurse network databases, HCP were asked to complete online questionnaire collecting information on perceptions of IBD and travel, confidence at providing travel advice, and the content of that advice.

Results 136 IBD patients (67[49\%] Crohn's disease, 60[44\%] male, median age 38 years[range 18-85]) and 105 HCP (98/105 [93\%] nurse specialists, 6/105[6\%] consultant, 1/105[1\%] registrar) responded. 85\%[106/136] patients report feeling adequately prepared for travel, although only 24\%[32/136] seek travel medical advice of any kind and only 11\%[15/136] from the IBD team; all despite $60 \%[82 / 136]$ reporting their IBD affected travel. Despite recommendations, only 4\%[5/136] had been prescribed antibiotics for self-medication of travellers diarrhoea. 52\%[36/69] of immunosuppressed patients are unaware they should avoid live vaccines. 39\%[53/136] patients buy travel insurance covering IBD, $70 \%[37 / 53]$ of which pay a premium. $70 \%[74 / 105]$ HCP felt IBD might limit travel in patients. $70 \%$ [74/105] HCP are confident giving travel advice, but 51\%[38/ 74] refer them to a travel clinic. $90 \%[94 / 105]$ are confident giving advice on travellers diarrhoea, but only 54\%[57/105] on vaccinations and 40\%[42/105] on insurance.

Conclusion Patients travel is affected by IBD, however, few seek expert medical advice prior to travel. HCP agree IBD affects travel and a majority are confident giving limited advice. It is concerning $52 \%$ of immunosuppressed patients are unaware they should avoid live vaccines, and only 54\% of HCP are 
confident giving advice on vaccinations. Results support the need for further travel specific research and better education in both groups.

\section{REFERENCES}

1 Soonwala et al. Inflamm Bowel Dis 2012;18(11):2079-85

2 Rahier et al. Journal of Crohn's and Colitis 2009:3(2):47-91

3 Wasan et al. Inflamm Bowel Dis 2014;20(2):246-50

4 Wasan et al. Inflamm Bowel Dis 2011;17(12):2536-60

Disclosure of Interest None Declared.

\section{PTU-093 AN EVALUATION OF AN IBD ADVICE SERVICE: IS IT MEETING ITS SERVICE AIMS?}

${ }^{1} \mathrm{G}$ Collins, ${ }^{2} \mathrm{M}$ Sastrillo, ${ }^{2} \mathrm{~J}$ Duncan*. 'Gastroenterology and Liver Services, Concord Repatriation General Hospital, Sydney, Australia; ${ }^{2}$ Department of Gastroenterology, Guy's and St Thomas' NHS Foundation Trust, London, UK

\subsection{6/gutjnl-2014-307263.167}

Introduction The provision of a dedicated and accessible IBD advice service (AS) is a key element of IBD management and, often, the responsibility of the Advanced, or specialist, IBD Nurse according to the N-ECCO Consensus statements. UK IBD Standards require IBD patients to have rapid access to specialist advice before the end of the next working day (EONWD). Our AS aims to provide timely access to clinical advice, support and acts as a point of contact to co-ordinate the patient journey. We evaluated if our advice service was meeting these goals.

Methods Over a 5 week period (23 working days) during October and November 2013, all contacts to the AS of a central London tertiary IBD service were recorded. Patients either called and left a message on an answering machine, or emailed a dedicated email address. Two experienced IBD CNS' collected data during each encounter. This included demographics of gender, age, and diagnosis; the format of contact (phone/email); if a medical opinion (IBD specialist or IBD registrar/fellow) was sought; time to response, and amount of time spent on each contact. The content of the encounter (administrative, clarification, a new query, or a symptomatic change/flare) was documented along with the response (administrative, information, results, treatment changes, medical decision), and the follow up required for the patient (routine, earlier or urgent outpatient appointment, or hospital admission/presentation to AandE).

Results 262 contacts were made to the AS. 4 could not be recontacted and 23 had missing data, leaving 235 complete encounters for analysis, of which 3 enquiries were non-IBD related. Those who contacted the AS were predominantly female (148/235, 62.98\%), between $26-35$ (97/235, 41.28\%), with a diagnosis of Crohn's Disease (160/235, 68.09\%), the latter reflecting the tertiary nature of our IBD service. 99.15\% (233/ 235 ) of contacts were replied to by EONWD, with 38.29\% (90/ $235)$ answered within $12 \mathrm{~h}$. The majority of contacts $(85.11 \%)$ were for clinical reasons with $14.89 \%$ administrative $(35 / 235)$. $51 / 235$ (21.70\%) pertained to flares. $88.94 \%$ (209/235) were autonomously handled by the IBD CNS though IBD Consultant/ Fellow support was required in 26 cases. AandE presentation was recommended to 2 patients $(2 / 235,0.85 \%)$ and $25(10.64 \%)$ had their outpatient appointment brought forward, meaning the vast majority were clinically managed without the need for additional outpatient review.

Conclusion Our IBD advice service provides patients with rapid access to specialist advice, symptom management and disease-specific information, meeting UK national standards. The IBD CNS' expertise means clinical enquiries can be effectively managed whilst avoiding additional, unnecessary burden to the patient and to outpatient clinics.

Disclosure of Interest None Declared.

\section{PTU-094 DO WE NEED POST INFLIXIMAB INFUSION MONITORING?}

K Robinson*, A Wright, M McAlindon, A Lobo. Gastroenterology, Royal Hallamshire Hospital, Sheffield, UK

\subsection{6/gutjnl-2014-307263.168}

Introduction Infliximab is used in the treatment of inflammatory bowel disease. It is administered as an intravenous infusion over $2 \mathrm{~h}$ with a $2 \mathrm{~h}$ monitoring period. Accelerated infusions have been shown to be safe and well tolerated, ${ }^{1}$ reducing nursing time and increasing patient satisfaction. ${ }^{2}$ It has been suggested that post infusion monitoring may not be necessary, ${ }^{3}$ and it was our aim to establish this.

Methods 310 infusions were administered to 103 patients over 6 months (January to July 2013). Infusions 1-4 were administered over $2 \mathrm{~h}$ with $2 \mathrm{~h}$ monitoring, 5-9 over $1 \mathrm{~h}$ with $1 \mathrm{~h}$ monitoring, and 10 onwards over 30 mins with no monitoring.

A reaction was classified as mild if no action was required and severe if symptoms required immediate action or treatment withdrawal. A drop in systolic BP of $\geq 20 \mathrm{~mm} / \mathrm{Hg}$ was recorded. Treatment of reaction and outcome were documented, including occurrence during or post infusion. Details of any delayed reactions post discharge were obtained from patient notes.

Results Of 41 patients receiving infusions 1-4, 2 patients $(4.87 \%)$ had an infusion reaction. One mild, and one severe. Both occurred during the first infusion. Both had previously been treated with infliximab.

In 35 patients receiving infusions 5-9, 1 patient (2.86\%) experienced a mild reaction during infusion 7 , then a severe reaction during infusion 9 .

No infusion reactions were observed during infusions $10+$ (122 infusions in 37 patients). 11 patients had infusions $10+$ over 1-2 $\mathrm{h}$ due to side effects with accelerated infusions or $10 \mathrm{mg} / \mathrm{kg}$ dose. These patients were not monitored post infusion.

One patient was hospitalised due to a delayed reaction one week after infusion 1 (previous infliximab treatment $108 \mathrm{~m}$ ). No side effects were observed during the infusion or monitoring period.

No reactions were recorded during the monitoring period in any of the treatment groups. One patient had a drop in systolic BP $(22 \mathrm{mg} / \mathrm{Hg})$ during the monitoring period of their $5^{\text {th }}$ infusion. No action was taken and the patient was discharged.

Conclusion This audit has demonstrated that post infliximab monitoring is not necessary. We estimate that this would save $494 \mathrm{~h}$ of patient and nurse time per annum at our centre.

\section{REFERENCES}

1 Donnellan CF, et al. Accelerated infliximab infusions are safe and well tolerated in patients with inflammatory bowel disease. Eur J Gastroenterol Hepatol 2009;21(1):71-75

2 Saxena $P$, et al. Safety and cost benefit of an accelerated infliximab infusion protocol in the treatment of ambulatory patients with inflammatory bowel diseases. Expert Opin Biol Ther 2013 Dec 21 [Epub ahead of print]

3 Bhat $\mathrm{S}$, et al. Are accelerated infliximab infusions safe in patients with inflammatory bowel disease? Inflammatory Bowel Disease 2010 Nov, 16 11;1922-5

Disclosure of Interest None Declared. 